\title{
Commentary: Association of Anemia with Venous Thromboembo- lism in Acutely III Hospitalized Patients: An APEX Trial Substudy
} Anmol Pitliya', Asrar Ahmad², Husnain Shaukat', Eiman Ghaffarpasand', Sadaf Sharfaei', Farima Kahe1, Syed Hassan A. Kazmi ${ }^{1}$, Gerald Chi ${ }^{*}$

'Division of Cardiovascular Medicine, Department of Medicine, Beth Israel Deaconess Medical Center, Harvard Medical School, Boston, Massachusetts, USA ${ }^{2}$ Department of Medicine, Abington Memorial Hospital, Abington, Pennsylvania, USA

\section{Article Info}

\section{Article Notes}

Received: May 23, 2018

Accepted: June 18, 2018

\section{${ }^{*}$ Correspondence:}

Dr. Gerald Chi, Division of Cardiovascular Medicine,

Department of Medicine,

Beth Israel Deaconess Medical Center, Harvard Medical School, 930 Commonwealth Avenue \#3,

Boston, Massachusetts 02215, USA; Telephone No: (617) 975-9952; Fax No: (617) 975-9955;

Email: geraldcchi@gmail.com

C $2018 \mathrm{Chi}$ G. This article is distributed under the terms of the Creative Commons Attribution 4.0 International License.

\section{ABSTRACT}

Anemia, commonly defined as hemoglobin concentration $<12.0 \mathrm{~g} /$ $\mathrm{dL}$ in women and $<13.0 \mathrm{~g} / \mathrm{dL}$ in men, is a prevalent condition that has been linked to unfavorable prognosis among hospitalized patients. Populationbased studies report that low hemoglobin level is associated with various thrombotic disorders including myocardial infarction, stroke, and venous thromboembolism (VTE). A recent study on acutely ill hospitalized patients extends the previous findings and demonstrates that anemic patients were at a greater risk for symptomatic VTE through 77 days of follow-up. Specifically, decreased hemoglobin concentration was associated with a two-fold risk of symptomatic DVT or non-fatal PE, despite the provision of pharmacologic thromboprophylaxis with enoxaparin or betrixaban, an FDA approved direct oral anticoagulant for VTE prevention. Additionally, hemoglobin measurement improved the risk discrimination and reclassification of a well-validated VTE assessment tool (i.e., International Medical Prevention Registry on Venous Thromboembolism [IMPROVE] VTE risk score). Based on these results, future studies should evaluate the use of laboratory biomarkers in conjunction with clinical variables to refine individualized VTE risk assessment.

\section{Abbreviations}

DVT, deep vein thrombosis

IMPROVE, International Medical Prevention Registry on Venous Thromboembolism

PE, pulmonary embolism

VTE, venous thromboembolism

According to the World Health Organization definition, anemia is a condition in which the number of red blood cells (and consequently their oxygen-carrying capacity) is insufficient to meet the body's physiologic needs ${ }^{1}$. Anemia is a prevalent condition among elderly patients and has been associated with unfavorable prognosis such as decreased physical performance, frequent hospitalization, and increased mortality ${ }^{2-4}$. Furthermore, accruing evidence suggests that anemic patients have a substantial long-term risk of arterial and venous thrombotic disorders ${ }^{5-8}$. Although the exact pathophysiology remains elusive, several mechanisms have been postulated for the association of quantitative and qualitative erythrocyte defects with thrombosis9. First, in anemic state, hypoxia-induced changes in gene expression may affect the interaction between erythrocytes and endothelium and therefore increase the risk for 
thrombosis ${ }^{10}$. Second, iron deficiency, the most common type of anemia globally, ${ }^{11}$ has been linked to thrombosis secondary to reduced inhibition of thrombopoiesis and subsequent reactive thrombocytosis ${ }^{12}$. Third, anemia may be accompanied by altered erythrocyte morphology (e.g., sickle cell disease [SCD] or hereditary spherocytosis) that increases blood viscosity ${ }^{13}$. Furthermore, increased erythrocyte phosphatidylserine exposure observed in SCD has been correlated with elevated circulating prothrombin fragment, indicating that erythrocytes in certain settings may potentiate thrombin generation ${ }^{14,15}$. Also, hemoglobin and adenosine diphosphate released from hemolyzed erythrocytes may enhance thrombosis by promoting platelet activation and aggregation ${ }^{16-18}$. Last, in aplastic anemia, T-cell mediated myelosuppression and cytokines such as tumor necrosis factor alpha, interleukin (IL)-6, and IL-2 may mediate the development of VTE, ${ }^{19}$ possibly by inducing the production of reactive oxygen species, which activates inflammatory processes and various transcription factors ${ }^{20}$. However, whether anemia would be associated with a short-term thrombotic risk independent of anticoagulation has not been comprehensively studied.

Recently,asub-study fromtheAPEXtrial(Acute Medically Ill Venous Thromboembolism Prevention with Extended Duration Betrixaban Trial; ClinicalTrials.gov identifier: NCT01583218) evaluated the relationship between hemoglobin and the risk of venous thromboembolism (VTE) among patients hospitalized for an acute medical illness $^{21}$. A total of 7513 hospitalized medical patients were randomized to receive thromboprophylaxis with either betrixaban for 35 to 42 days or standard-of-care enoxaparin for $10 \pm 4$ days and followed for 77 days. Baseline hemoglobin measurement was performed in 6861 patients at a central laboratory, with the normal reference range of 12.5 to $17.0 \mathrm{~g} / \mathrm{dL}$ for males and 11.0 to $15.5 \mathrm{~g} /$ $\mathrm{dL}$ for females. The endpoint of the study was symptomatic VTE, defined as a composite of symptomatic deep vein thrombosis (DVT), non-fatal pulmonary embolism (PE), or VTE-related mortality. VTE events were adjudicated by an independent clinical events committee blinded to treatment allocation. The relationship between anemia and VTE events was first evaluated by fitting a univariable and multivariable logistic regression model composed of thromboprophylaxis and VTE risk factors. To further assess the association between baseline hemoglobin levels and symptomatic VTE events, hemoglobin levels were coded as an ordinal variable and VTE event rate across hemoglobin levels was performed using the CochranArmitage test for trend. In addition, a locally weighted regression smoothing approach was applied to evaluate the dose-dependent relationship between hemoglobin concentration (as a continuous variable) and VTE risk, with adjustment for covariates identified in the multivariable analysis (i.e., D-dimer, thromboprophylaxis, history of previous VTE, and intensive or coronary care unit stay). Incremental prognostic value of hemoglobin measurement was evaluated in the International Medical Prevention Registry on Venous Thromboembolism [IMPROVE] VTE risk assessment model by computing the improvement in area under the receiver- operating-characteristic curve $(\triangle \mathrm{AUC})$, integrated discrimination improvement (IDI), and net reclassification improvement (NRI) ${ }^{22}$.

Compared with non-anemic individuals, anemic patients had a two-fold increased risk of symptomatic VTE ( $R R=1.94$ [95\% CI: 1.27-2.98]; p=0.002), symptomatic DVT $(R R=2.29$ [1.12-4.68]; $\mathrm{p}=0.019)$, and non-fatal PE $(R R=2.63$ [1.22-5.65]; $p=0.010)$. After accounting for thromboprophylaxis and other VTE risk factors (e.g., history of VTE, current lower limb paralysis, active cancer, intensive or coronary care unit stay, and age $>60$ years), anemia remained associated with symptomatic VTE (adjusted OR=1.71 [1.09-2.69]; p=0.020). There was an inverse dose-response relationship between baseline hemoglobin concentration and the estimated probability of VTE. Moreover, anemia significantly enhanced VTE risk discrimination and reclassification when incorporated in the IMPROVE VTE risk score. Specifically, adding hemoglobin to the IMPROVE score increased its AUC by 0.049 (95\% CI: 0.012-0.086; $\mathrm{p}=0.0096)$, with a modest increase in IDI of 0.0015 (0.0001-0.0029; p=0.0399). The continuous NRI and categorical NRI were 0.257 (95\% CI: 0.066-0.448; p=0.0132) and 0.327 (95\% CI: 0.162-0.492; p=0.0001), respectively. Improvement in VTE risk reclassification was primarily driven by the nonevent group (i.e., using hemoglobin to exclude the risk of symptomatic VTE).

Previously it has been shown that the risk of VTE and related rehospitalization extends beyond the standard course of anticoagulation and may continue for several months ${ }^{23}$. Furthermore, more than half of the VTE events are associated with a recent or current hospitalization despite near-universal in-hospital VTE prophylaxis ${ }^{24}$. In corroboration with previous reports, ${ }^{25,26}$ the presence of anemia at hospital admission may adversely impact both short- and long-term clinical outcome by predisposing patients to thrombotic disorders. Whilst the use of extended-duration betrixaban for thromboprophylaxis in acute medically ill patients may not reduce all-cause mortality, ${ }^{27}$ it was associated with a lower rate of VTErelated mortality when compared to standard-duration enoxaparin $^{28}$. This may be explained by the prevalent comorbidities among the study population that obscured the survival benefit but not disease-specific benefit (i.e., reduction in VTE-related death). Additionally, patients who received extended-duration betrixaban had a decreased incidence of fatal or irreversible events (a composite of safety [fatal bleeding or intracranial hemorrhage] and 
efficacy [cardiopulmonary death, myocardial infarction, pulmonary embolism, and ischemic stroke] endpoints) compared with standard-duration enoxaparin ${ }^{29}$.

Although the causes of anemia were not determined in the APEX trial, low hemoglobin level may reflect an underlying disorder (such as nutritional deficiency, chronic inflammation, or cancer and related chemotherapy) that contributes to the increased incidence of VTE. Hemoglobin level may, therefore, be considered as an integrative measure of multiple pathologic processes in medical inpatients. Another plausible mechanism is that anemic patients may be more likely to have endothelial dysfunction, ${ }^{30-32}$ blood stasis, ${ }^{13}$ and/or hypercoagulability ${ }^{33,34}$-namely the Virchow's triad-and an increased risk of thrombosis ${ }^{35,36}$. Regardless of whether anemia is mechanistically related to thrombosis or an index of disease burden, these results support the use of hemoglobin level in refining risk assessment among hospitalized patients despite thromboprophylaxis provision.

The strength of the study resides in several aspects. In contrast with population-based studies or registries that assess the long-term risk of thrombotic diseases, the present study possesses direct clinical implications as the proximate risk of VTE was rigorously ascertained through 77 days. This time window encompasses the post-hospital discharge period in which patients remain at a substantial VTE risk ${ }^{37}$. Furthermore, hemoglobin measurement is of low cost, widely available, and routinely utilized in clinical practice as part of the complete blood counts when evaluating acutely ill hospitalized patients. In addition, the analysis accounts for potential confounding effect by the provision of thromboprophylaxis as well as other VTE risk factors, although the potential confounding effect of severe lung disease was not reported. However, it is noteworthy that given the exploratory nature of the study, the findings indicate the correlation rather than causation between anemia and VTE. These findings reinforce the prognostic value of hemoglobin measurement, alone or in combination with VTE risk assessment model. Future research is warranted to investigate the use of hematologic tests or other biomarkers in conjunction with clinical variables to refine individualized VTE prediction ${ }^{38}$.

\section{Disclosure}

Dr. Chi received research grant support paid to the Beth Israel Deaconess Medical Center from Portola, Bayer, and Janssen Research. All remaining authors report no conflicts of interest.

\section{References}

1. Haemoglobin concentrations for the diagnosis of anaemia and assessment of severity. Vitamin and Mineral Nutrition Information System Geneva, World Health Organization. Vol. (WHO/NMH/NHD/ MNM/11.1); 2011.
2. Culleton BF, Manns BJ, Zhang J, et al. Impact of anemia on hospitalization and mortality in older adults. Blood. 2006; 107(10): 3841-3846.

3. Penninx BW, Pahor M, Woodman RC, et al. Anemia in old age is associated with increased mortality and hospitalization. The Journals of Gerontology Series A: Biological Sciences and Medical Sciences. 2006; 61(5): 474-479.

4. Penninx BW, Guralnik JM, Onder G, et al. Anemia and decline in physical performance among older persons. The American journal of medicine. 2003; 115(2): 104-110.

5. Nikolsky E, Aymong ED, Halkin A, et al. Impact of anemia in patients with acute myocardial infarction undergoing primary percutaneous coronary intervention: analysis from the Controlled Abciximab and Device Investigation to Lower Late Angioplasty Complications (CADILLAC) Trial. Journal of the American College of Cardiology. 2004; 44(3): 547-553.

6. Pilgrim T, Vetterli F, Kalesan B, et al. The impact of anemia on longterm clinical outcome in patients undergoing revascularization with the unrestricted use of drug-eluting stents. Circulation: Cardiovascular Interventions. 2012; 5(2): 202-210.

7. Hung $\mathrm{SH}$, Lin $\mathrm{HC}$, Chung SD. Association between venous thromboembolism and iron-deficiency anemia: a population-based study. Blood Coagulation \& Fibrinolysis. 2015; 26(4): 368-372.

8. Lin CL, Lin CL, Tzeng SL, et al. Aplastic anemia and risk of deep vein thrombosis and pulmonary embolism: A nationwide cohort study. Thrombosis research. 2017; 149: 70-75.

9. Byrnes JR, Wolberg AS. Red blood cells in thrombosis. Blood. 2017; 130(16): 1795-1799.

10. Sergueeva A, Miasnikova G, Shah BN, et al. Prospective study of thrombosis and thrombospondin-1 expression in Chuvash polycythemia. haematologica. 2017; 102(5): e166-e169.

11. Kassebaum NJ, Jasrasaria R, Naghavi M, et al. A systematic analysis of global anemia burden from 1990 to 2010. Blood. 2014; 123(5): 615624.

12. Franchini $M$, Targher $G$, Montagnana $M$, et al. Iron and thrombosis. Ann Hematol. 2008; 87(3): 167-173.

13. Piety NZ, Reinhart WH, Pourreau PH, et al. Shape matters: the effect of red blood cell shape on perfusion of an artificial microvascular network. Transfusion. 2016; 56(4): 844-851.

14. Whelihan M, Lim M, Mooberry M, et al. Thrombin generation and cell-dependent hypercoagulability in sickle cell disease. Journal of Thrombosis and Haemostasis. 2016; 14(10): 1941-1952.

15. Setty BY, Rao AK, Stuart MJ. Thrombophilia in sickle cell disease: the red cell connection. Blood. 2001; 98(12): 3228-3233.

16. Rother RP, Bell L, Hillmen P, et al. The clinical sequelae of intravascular hemolysis and extracellular plasma hemoglobin: a novel mechanism of human disease. Jama. 2005; 293(13): 1653-1662.

17. Reimers RC, Sutera SP, Joist JH. Potentiation by red blood cells of shear-induced platelet aggregation: relative importance of chemical and physical mechanisms. Blood. 1984; 64(6): 1200-1206.

18. Santos M, Valles J, Marcus A, et al. Enhancement of platelet reactivity and modulation of eicosanoid production by intact erythrocytes. A new approach to platelet activation and recruitment. The Journal of clinical investigation. 1991; 87(2): 571-580.

19. Lin CL, Tzeng SL, Chung WS. Aplastic anemia and risk of deep vein thrombosis and pulmonary embolism: A nationwide cohort study. Thromb Res. 2017; 149: 70-75.

20. Li YD, Ye BQ, Zheng SX, et al. NF-kappaB transcription factor p50 critically regulates tissue factor in deep vein thrombosis. J Biol Chem. 2009; 284(7): 4473-4483. 
21. Chi G, Gibson CM, Hernandez AF, et al. Association of Low Hemoglobin with Symptomatic Venous Thromboembolism in Acutely Il Hospitalized Medical Patients: an APEX Trial Substudy. The American journal of medicine. 2018.

22. Spyropoulos AC, Anderson FA, FitzGerald G, et al. Predictive and associative models to identify hospitalized medical patients at risk for VTE. Chest. 2011; 140(3): 706-714.

23. Chi G, Yee MK, Amin AN, et al. Extended-Duration Betrixaban Reduces the Risk of Rehospitalization Associated With Venous Thromboembolism Among Acutely Ill Hospitalized Medical Patients: Findings From the APEX Trial (Acute Medically Ill Venous Thromboembolism Prevention With Extended Duration Betrixaban Trial). Circulation. 2018; 137(1): 91-94.

24. Chi G, Gibson CM, Hernandez AF, et al. Association of Anemia with Venous Thromboembolism in Acutely Ill Hospitalized Patients: An APEX Trial Substudy. Am J Med. 2018 Apr 13. pii: S00029343(18)30312-7. doi: 10.1016/j.amjmed.2018.03.031.

25. Young JB, Abraham WT, Albert NM, et al. Relation of low hemoglobin and anemia to morbidity and mortality in patients hospitalized with heart failure (insight from the OPTIMIZE-HF registry). American Journal of Cardiology. 2008; 101(2): 223-230.

26. Sabatine MS, Morrow DA, Giugliano RP, et al. Association of hemoglobin levels with clinical outcomes in acute coronary syndromes. Circulation. 2005; 111(16): 2042-2049.

27. Cohen AT, Harrington RA, Goldhaber SZ, et al. Extended Thromboprophylaxis with Betrixaban in Acutely Ill Medical Patients. N Engl J Med. 2016; 375(6): 534-544.

28. Gibson CM, Nafee T, Goldhaber SZ, et al. Abstract 20393: FullDose Betrixaban Reduces Venous Thromboembolism-Related Mortality: An APEX Trial Substudy. Circulation. 2017; 136(Suppl 1): A20393-A20393.
29. Gibson CM, Korjian S, Chi G, et al. Comparison of Fatal or Irreversible Events With Extended-Duration Betrixaban Versus Standard Dose Enoxaparin in Acutely Ill Medical Patients: An APEX Trial Substudy. Journal of the American Heart Association. 2017; 6(7).

30. Solovey A, Lin Y, Browne P, et al. Circulating activated endothelial cells in sickle cell anemia. N Engl J Med. 1997; 337(22): 1584-1590.

31. Hebbel RP. Ischemia-reperfusion injury in sickle cell anemia: relationship to acute chest syndrome, endothelial dysfunction, arterial vasculopathy, and inflammatory pain. Hematol Oncol Clin North Am. 2014; 28(2): 181-198.

32. Yildiz A, Oflaz H, Pusuroglu $\mathrm{H}$, et al. Left ventricular hypertrophy and endothelial dysfunction in chronic hemodialysis patients. Am J Kidney Dis. 2003; 41(3): 616-623.

33. Vannucchi AM, Barbui T. Thrombocytosis and thrombosis. Hematology Am Soc Hematol Educ Program. 2007: 363-370.

34. Ataga KI, Cappellini MD, Rachmilewitz EA. Beta-thalassaemia and sickle cell anaemia as paradigms of hypercoagulability. $\mathrm{Br} J$ Haematol. 2007; 139(1): 3-13.

35. Stone J, Hangge P, Albadawi $\mathrm{H}$, et al. Deep vein thrombosis: pathogenesis, diagnosis, and medical management. Cardiovasc Diagn Ther. 2017; 7(Suppl 3): S276-S284.

36. Kumar DR, Hanlin E, Glurich I, et al. Virchow's contribution to the understanding of thrombosis and cellular biology. Clin Med Res. 2010; 8(3-4): 168-172.

37. Spyropoulos AC, Raskob GE. New paradigms in venous thromboprophylaxis of medically ill patients. Thrombosis and haemostasis. 2017; 117(09): 1662-1670.

38. Gibson CM, Spyropoulos AC, Cohen AT, et al. The IMPROVEDD VTE risk score: incorporation of D-dimer into the IMPROVE score to improve venous thromboembolism risk stratification. TH Open. 2017; 1(01): e56-e65. 